\title{
Pills or public health?
}

\author{
Fiona Godlee editor in chief
}

The BMJ

How best to tackle the rising tide of non-communicable disease linked to lifestyle, or, more accurately, linked to the environments in which people live? With pills or with traditional public health interventions: healthier food and cleaner air?

The push for pills is strong. As reported by the Science Media Centre, the expert response to NICE's draft guideline on hypertension has been glowing (http://bit.ly/2IjMEgD). NICE wants to lower the threshold for starting treatment for mild hypertension. All six experts (of whom two declare industry ties and three give no statement of interests at all) welcome the draft guidance. One expert, also quoted in our own news report (doi:10.1136/bmj.11105), suggests the guidance doesn't go far enough.

Our editorialists this week take a different view (doi:10.1136/ bmj.11657). (In the interests of transparency, we should say that one of them, John Brodersen, is on the organising committee of the Preventing Overdiagnosis conference (preventingoverdiagnosis.net)). They say the draft guidance focuses too narrowly on treatment and on the views of health professionals rather than patients; that it fails to see the wider impact of capturing millions of healthy people in its diagnostic net (doi:10.1136/bmj.k2357); that it understates the harms not only of treatment but also of being labelled as hypertensive; and that it adopts an arbitrary cut off without adequate discussion of the importance of patients' preferences and values. These views chime with a study recently summarised in our NIHR Signals series (doi:10.1136/bmj.1571). Luckily, there is still time for NICE to think again, especially as there is now a handy checklist of items for guideline panels to consider before changing the definition of a disease (doi:10.1136/bmjebm-2018111148).

Further push back on pills this week comes from Nigel Hawkes (doi:10.1136/bmj.11779). Surprised by claims in the press that giving statins to everyone aged over 75 would save 8000 lives a year in the UK, we asked him to investigate. The 8000 figure came from the Cholesterol Treatment Trialists' Collaboration, whose meta-analysis was published in the Lancet in February. Hawkes, a longstanding contributor to The $B M J$, is not overly impressed. Potential lives saved are the currency of people backing statins, he says, but they are meaningless to patients. It's better to provide numbers needed to treat or average years of life saved. Sadly, these give rather less newsworthy numbers. By comparison, the UK government's programme to cut sugar in food could, if properly implemented, significantly reduce levels of obesity, conclude Ben Amies-Cull and colleagues (doi:10.1136/bmj.11417), with benefit to physical and mental health. But it must be part of a wider package of measures, says Annalijn Conklin in the linked editorial (doi:10.1136/bmj.11705), and the government will have to keep the industry's feet to the fire. 